\section{Inflammatory pseudotumor of the spleen}

\author{
Georgia McMahon, ${ }^{1}$ Kirsty Rady, ${ }^{2}$ \\ Henry Miles Prince ${ }^{2}$
}

1Peninsula Health, Frankston Hospital, Victoria; ${ }^{2}$ Centre of Blood Cell Therapies, Peter MacCallum Cancer Centre, Melbourne, Victoria, Australia

\begin{abstract}
Isolated splenic inflammatory pseudotumors (IPT) are extremely rare, typically benign, inflammatory lesions with varied clinical presentations that pose a diagnostic challenge to clinicians due to their similarity in appearance to neoplasms. We present the case of a young woman diagnosed with a splenic IPT following investigation for persistent anemia, raised inflammatory markers, and polyclonal hypergammaglobulinemia, whose symptoms resolved completely following splenectomy. This case highlights the need to consider this diagnosis when evaluating patients with a splenic mass of unknown etiology.
\end{abstract}

\section{Introduction}

Splenic tumors are rare and can be caused by a wide range of benign and malignant conditions including cysts and abscesses, granulomatous processes, Castleman's disease, angiosarcoma, teratoma, lymphoma, and metastatic carcinoma. ${ }^{1-6}$ Inflammatory pseudotumors (IPT) are uncommon, yet important lesions to consider in the differential diagnosis of splenic masses. ${ }^{1-3}$ These tumor-like masses of inflammatory cells, occur in a number of different organ systems, with the most common being the lung and orbit; however they have also been reported in the meninges, testis, gastrointestinal tract, heart, liver, bladder, thyroid, nasal cavity and soft tissue. ${ }^{1-3,5-7}$ Primary splenic IPT is rare; and the precise number of cases is uncertain, with variable numbers reported in the literature since its first description in 1984 by Cotelingam and Jaffe. ${ }^{1-5,7,8}$ The number of cases reported varies between 80 reported in $2008,1,5,6$ to 114 in the most recent studies, ${ }^{4}$ however, the available data is incomplete, and it remains unclear whether all of these cases are primary solitary splenic IPT or whether some of these are cases of secondary splenic involvement, or misdiagnoses of other histologically similar lesions.

Inflammatory pseudotumors may present with a wide range of clinical symptoms, non- specific imaging findings, and often, features of an underlying systemic inflammatory response, making the diagnosis challenging. ${ }^{8}$ The chronic inflammatory response is characterized biochemically by raised inflammatory markers, and often, polyclonal hypergammaglobulinemia, resulting from a diffuse activation of plasma cells. Polyclonal hypergammaglobulinemia is seen in chronic infections and inflammatory conditions, connective tissue diseases, cases of liver disease, and ultimately, a myriad of other conditions leading to chronic stimulation of the immune response. ${ }^{9}$

We present a case of a young woman with non-specific, chronically elevated inflammatory markers, including raised $\mathrm{C}$-reactive protein (CRP), erythrocyte sedimentation rate (ESR) and polyclonal hypergammaglobulinemia, together with an isolated splenic lesion, who was found to have a rare inflammatory pseudotumor of the spleen.

\section{Case Report}

A 19-year-old woman was referred to our clinic for investigation of a 15-month history of fatigue, raised inflammatory markers, anemia and a polyclonal gammopathy of uncertain etiology. The patient originally presented to her general practitioner for a routine check-up prior to an extended overseas trip; she was well with no significant medical history, and asymptomatic of her anemia. Screening blood tests were suggestive of an iron deficiency anemia [hemoglobin $(\mathrm{Hb}) 105 \mathrm{~g} / \mathrm{L}$, mean corpuscle volume (MCV) $77 \mathrm{fL}$, ferritin (Fe) $2 \mathrm{umol} / \mathrm{L}$, transferrin saturation 3\%] associated with elevated inflammatory markers ESR $73 \mathrm{~mm} / \mathrm{hr}$, CRP $45 \mathrm{mg} / \mathrm{L}$ and a low vitamin D level. Her total protein was $82 \mathrm{~g} / \mathrm{L}$ with a globulin level of $48 \mathrm{~g} / \mathrm{L}$; serum protein electrophoresis (SPEP) demonstrated a polyclonal response.

Numerous investigations and specialist consultations followed to identify the cause of this non-specific inflammatory response. Repeat blood tests including SPEP and hematinics were essentially unchanged; blood cultures, autoantibodies, and hemoglobin electrophoresis were unremarkable. Further investigations including a transthoracic echocardiogram, chest x-ray, magnetic resonance imaging (MRI) small bowel series, colonoscopy and gastroscopy were non-contributory. Initial treatment consisted of an iron infusion and commencement of daily vitamin D replacement.

Our patient continued with her plans to travel to England for a year. She had two followup blood tests while overseas, which again remained unchanged. On return to Australia, and in the setting of ongoing investigation for these persisting abnormalities, our patient developed anxiety and began to report a variety
Correspondence: Henry Miles Prince, Centre of Blood Cell Therapies, Peter MacCallum Cancer Centre, Locked Bag 1, A’Beckett St, Victoria 8006, Australia.

Tel.: +61.3965.61700.

E-mail: miles.prince@petermac.org

Key words: Splenic tumor; inflammatory pseudotumor; polyclonal gammopathy; benign splenic lesion; hypergammaglobulinemia.

Contributions: the authors contributed equally.

Conflict of interest: the authors declare no potential conflict of interest.

Received for publication: 15 March 2015.

Revision received: 4 May 2015.

Accepted for publication: 5 May 2015.

This work is licensed under a Creative Commons Attribution NonCommercial 3.0 License (CC BYNC 3.0).

(C) Copyright G. McMahon et al., 2015

Licensee PAGEPress, Italy

Hematology Reports 2015; 7:5905

doi:10.4081/hr.2015.5905

of non-specific symptoms, such as fatigue and muscle pains, which she attributed to her abnormal results. She was subsequently referred for a hematology opinion.

Once again, her hematology, electrolytes, hematinics, autoantibodies and SPEP were checked, and remained unchanged. Viral testing for Human HerpesVirus-8 (HHV-8) and Epstein-Barr virus (EBV) were negative. There was no evidence of lymphadenopathy or hepatosplenomegaly on physical examination. The patient was referred for a positive emission tomography-computed tomography (PETCT) scan for further investigation, and hopefully exclusion, of malignancy. Unexpectedly, the PET scan identified an area of mild fludeoxyglucose-18 (FDG) uptake in the superior pole of the spleen; this was verified on CT where a heterogeneous lesion with a small area of calcification was noted (Figure 1). On review of her prior MRI, conducted 13 months previously, a similar sized lesion was retrospectively identified (Figure 2). Examination of the bone marrow demonstrated reactive trilineage hematopoiesis with no evidence of lymphoma or plasma cell proliferation.

In view of the PET-avid splenic lesion, an uncomplicated, elective, laparoscopic splenectomy was performed for diagnostic and treatment purposes. On inspection, the spleen was partially morcelated, weighting 178 grams with no discrete lesion identified on serial sectioning. Microscopically, some sections showed replacement of splenic parenchyma by a lesion with abundant calcification and iron deposi- 
tion, which was composed of interlacing fascicles of haphazardly arranged spindled myofibroblasts with extensive hemorrhage, and a polymorphic inflammatory infiltrate comprising plasma cells, eosinophils, small mature lymphocytes, and hemosiderin-laden macrophages (Figure 3A). The adjacent spleen appeared normal with preserved red and white pulp. The myofibroblastic cells were strongly positive for SMA (Figure 3B) and negative for desmin, CD34, CD21, CD8, and ALK1. Additional stains show that the majority of spindled cells are negative for S100 protein, and no acid fast bacilli were seen with Ziehl-Neelsen and Wade Fite stains. In-situ hybridization for Epstein-Barr virus encoded-small RNA's (EBER) and HHV8 were negative. A diagnosis of splenic IPT was made.

She had an uncomplicated post-operative course and pleasingly, all hematological and biochemical abnormalities resolved within two months post splenectomy. She remains well 11 months later.

\section{Discussion}

Inflammatory pseudotumors are rare masses of inflammatory cells that can be found in various anatomical areas of the body. ${ }^{4-7}$ The name inflammatory pseudotumor was first coined in 1954 by Umiker and Iverson, ${ }^{8,10}$ and encompasses a spectrum of inflammatory lesions including plasma cell granuloma, plasma cell pseudotumor and myofibroblastic tumor, which are all thought to arise from a similar underlying inflammatory disease process, eventually resulting in a tumor like mass of inflammatory cells. . $^{7,10}$

The pathogenesis and etiology of inflamma-

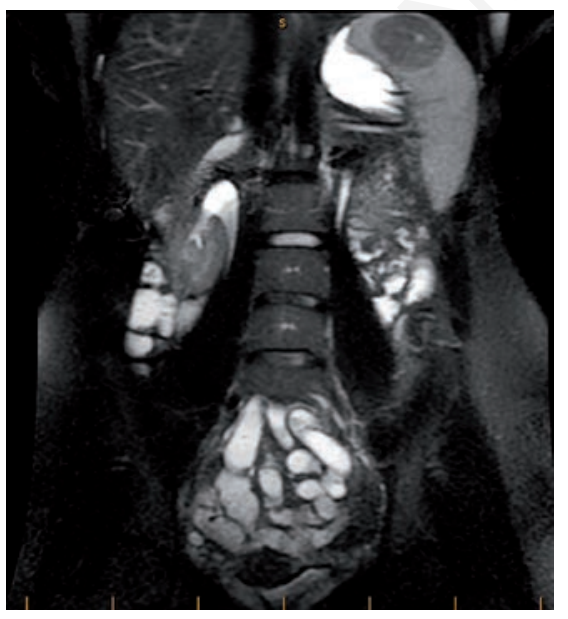

Figure 2. Magnetic resonance imaging of abdomen demonstrating a hypodense lesion on the superior pole of the spleen. tory pseudotumors is largely unknown and attracts varied theories according to the organ system involved, ${ }^{1,10}$ however, the two most widely accepted theories describe IPT as either pri- mary reactive and inflammatory processes or low-grade neoplastic processes with secondary inflammation. ${ }^{5}$ Splenic IPTs have not been reported to possess malignant properties, and

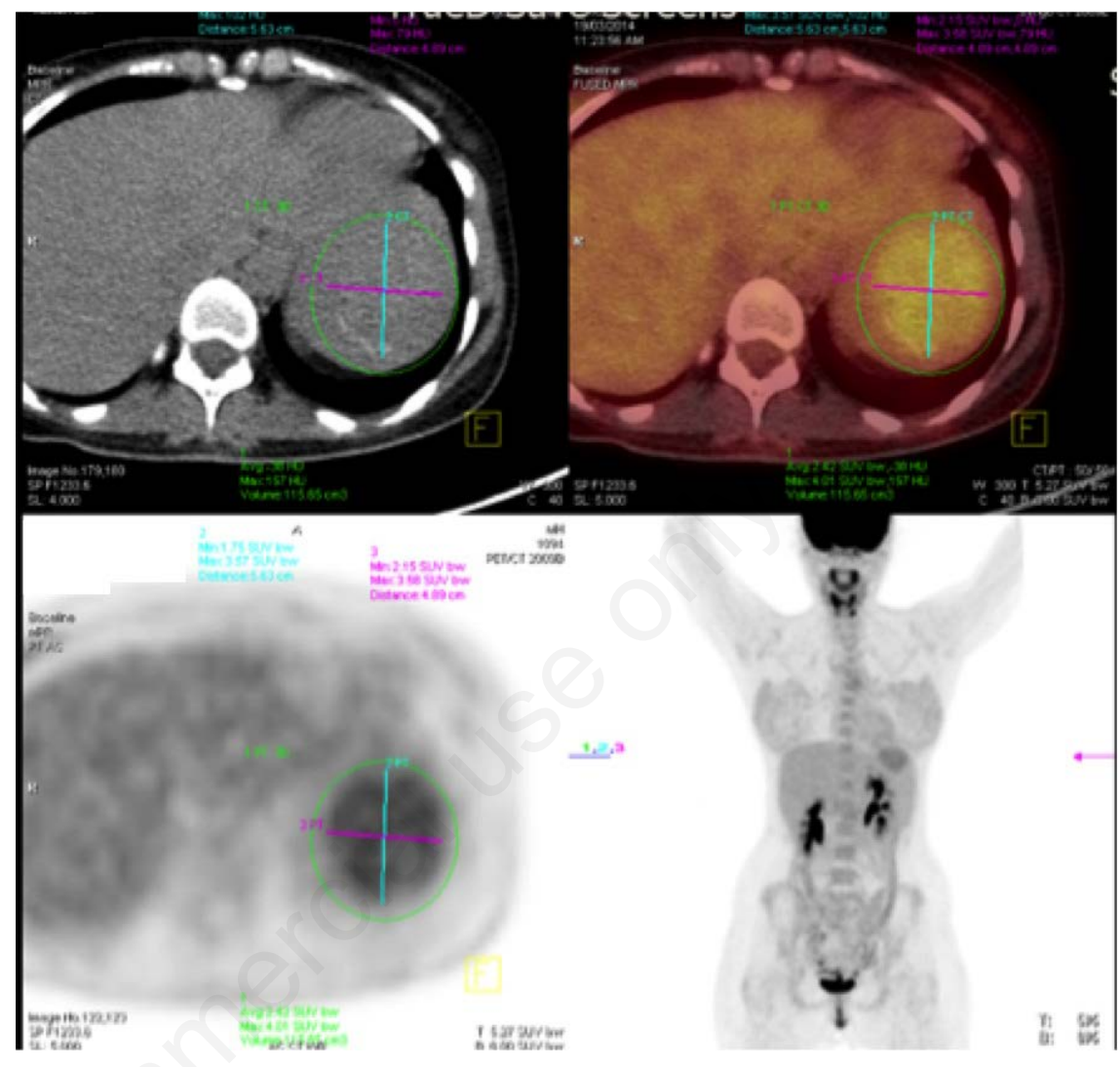

Figure 1. Positron emission tomography-computed tomography (PET-CT) scan of the abdomen highlighting a lesion with mild fludeoxyglucose-18 uptake on the superior pole of the spleen on PET, with CT verification of a heterogeneous lesion with an area of calcification.

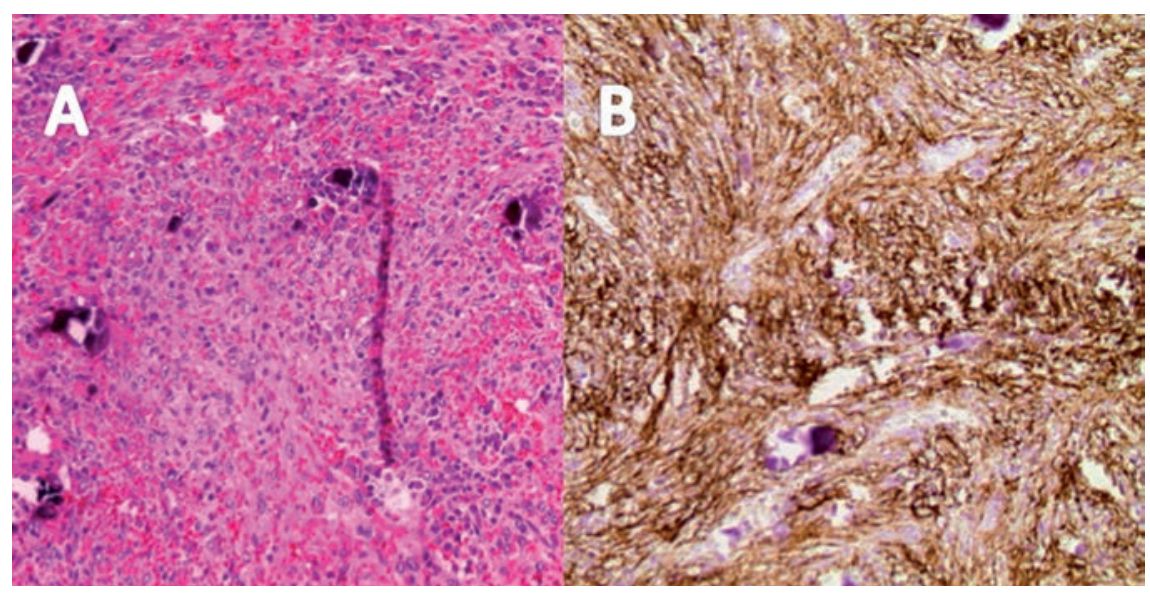

Figure 3. A) Hematoxylin and Eosin stain of splenic tissue demonstrating a lesion with haphazardly arranged fibroblasts with extensive hemorrhage, clusters of mature plasma cells, eosinophils and small numbers of lymphocytes. B) Smooth muscle actin stain demonstrating heavy fibroblastic component. 
have commonly been associated with both EBV and HHV- 8 viruses, ${ }^{1,2}$ and a review of all splenic IPT cases presented by Rajabi et al. ${ }^{3}$ confirmed $41.2 \%$ of all published cases of splenic IPTs tested positive EBV RNA. Other etiologies including trauma with secondary focal parenchymal necrosis, chronic infection, low-grade neoplastic processes, vascular causes and immunological derangement have been suggested. $1,2,4,6,10$ This is still a topic of discussion.

Many splenic IPTs are found incidentally, although presentations with left upper quadrant pain or epigastric pain, fever, weight loss and splenomegaly have been described.2,3,5,6,8,10 Many patients have no detectable laboratory abnormalities; ${ }^{2,10}$ however, anemia, thrombocytosis, hypercalcemia, both monoclonal and polyclonal hypergammaglobulinemia, and elevated inflammatory markers have been described. ${ }^{3-5,10}$ Based on case studies, splenic IPTs have been found in adults as young as 19, but are more common in those of middle to advanced age; there does not seem to be a gender association.2,6

The diagnosis of splenic ITP is challenging. Ultrasound, CT scan, MRI and PET scan are all commonly employed, but to date do not demonstrate many defining diagnostic characteristics allowing differentiation from other lesions. ${ }^{1,2,5,8,10}$ CT imaging commonly demonstrates a low-density mass in both nonenhanced and enhanced modes, while MRI shows low to isointensity on T1- weighted imaging, and high intensity with surrounding low intensity on T2- weighted images. MRI may be able to differentiate cavernous hemangioma, lymphangioma, hamartoma from IPT, based on the high intensity seen on T2-weighted images; however IPT mirrors malignant neoplasms and lymphoma in appearance on MRI. PET scan demonstrates FDG uptake within the IPT at a lower standardized uptake value (SUV) than malignant tumors, however variations in fibrosis and inflammation, and thus SUV, between IPTs mean this is of minimal use. Unfortunately, imaging alone is of limited value in differentiating IPT from other splenic lesions including benign inflammatory lesions, angiosarcoma, lymphoma and metastatic disease. ${ }^{1,2,5,8,10}$

Histological examination is required for diagnosis, and thus, splenectomy is the management of choice. ${ }^{2,10}$ Fine needle aspiration and core biopsy have been reported but are not recommended due to poor specificity, bleeding potential and risk of seeding if the tumor is malignant. ${ }^{2,5,6}$ In most cases of splenic IPT surgical management is deemed curative, and to the best of our knowledge there have been no reports of local invasion, recurrence or metastasis. ${ }^{2,5}$

Pathologically, IPTs resemble a benign tumor, comprising inflammatory cells and lymphoid cells with a well-circumscribed fibroblas- tic stroma. Histologically, the cellular composition can be extremely heterogeneous, with cells associated with both acute and chronic inflammation. They are characterized by proliferation of epitheloid cells, myofibroblastic spindle cells and collagen, mixed with morphologically normal lymphocytes, plasma cells, eosinophils and histiocytes. ${ }^{3,10}$

Splenic IPTs often share similarities to neoplasms and other inflammatory diseases, and need to be differentiated histologically with the addition of immunohistochemistry. Foremost, it is vital to distinguish IPT from IPT-like follicular dendritic cell sarcoma (IPT-like FDC sarcoma), an extremely rare lesion which has a similar histologic appearance to IPT, but can be more aggressive. ${ }^{11}$ Recognized as a variant of follicular dendritic cell sarcoma (FDC sarcoma), IPTlike FDC sarcoma has a female predilection, is almost always localized to the liver or spleen, and is associated with a prominent lymphoplasmacytic infiltrate. ${ }^{5}$ We acknowledge the difficulty in differentiating these lesions based on histopathology alone. From the limited available literature, IPT-like FDC sarcoma is classically CD21, CD23, CD35, CNA 42, and Clusterin positive on immunostaining, and EBV is almost always clonally expanded in the tumor cells. ${ }^{11,12}$ IPT-like FDC sarcomas show variable staining for SMA, HHF-35, S100 protein, and CD68, and they are usually ALK negative. $1^{1,12}$ The other major mesenchymal lesions in the spleen that should be excluded include splenic harmatoma, sclerosing angiomatoid nodular transformation (SANT), and age-related EBV-associated lymphoproliferative disorder; in these cases, the application of various FDC markers is also essential.

Based on the CD21, ALK1 and EBER negativity together with the diffusely positive SMA we are confident in our diagnosis of IPT; however, additional immunostaining for CD 35 and CD23 would have been beneficial. This case highlights the need for consideration of a wide range of diagnoses during initial investigation, and also the need for rigorous histopathologic study of the specimen to ensure the diagnosis is made conclusively. Given the difficultly in differentiation between IPT and IPT-like sarcoma, it is possible that a proportion of the previously reported 114 cases of splenic IPT were incorrectly diagnosed due to the lack of required immunohistochemical staining to sufficiently exclude IPT-like sarcoma.

\section{Conclusions}

Splenic IPTs are rare, usually benign, inflammatory lesions with varied clinical presentations that pose a diagnostic challenge to clinicians due to their similarity in appearance to neoplasms. This case serves as a reminder to consider this rare diagnosis when evaluating patients with a splenic mass of unknown etiology, and highlights the need to be meticulous in order to differentiate IPT histologically from other similar lesions, particularly IPT-like FDC sarcoma, through engagement of experienced morphologists and the use of immunohistochemistry.

\section{References}

1. Yarmohammadi H, Nakamoto D, Faulhaber PF, et al. Inflammatory pseudotumor of the spleen: review of clinical presentation and diagnostic methods. J Radiol Case Rep 2011;5:16-22.

2. Ma ZH, Tian XF, Ma J, Zhao YF. Inflammatory pseudotumor of the spleen: a case report and review of published cases. Oncol Lett 2013;5:1955-7.

3. Rajabi P, Noorollahi H, Hani M, Bagheri M. Inflammatory pseudotumor of spleen. Adv Biomed Res 2014;3:29.

4. Ugalde P, Garcia Bernardo C, Granero P, et al. Inflammatory pseudotumor of spleen: a case report. Int J Surg Case Rep 2015;7C:145-8.

5. Jarboui S, Hlel A, Bekkay MA, et al. Epstein-Barr virus positive inflammatory pseudotumor of the spleen: case report and review of the literature. Case Rep Clin Med 2014;3:443-9.

6. Yan J, Peng C, Yang W, et al. Inflammatory pseudotumour of the spleen: report of 2 cases and literature review. Can J Surg 2008;51:75-6.

7. Lewis JT, Gaffney RL, Casey MB, et al. Inflammatory pseudotumor of the spleen associated with a clonal epstein-barr virus genome: case report and review of the literature. Am J Clin Pathol 2003;120:56-61.

8. Patnana M, Sevrukov AB, Elsayes KM, et al. Inflammatory pseudotumor: the great mimicker. AJR Am J Roentgenol 2012;198: W217-27.

9. Dispenzieri A, Gertz MA, Therneau TM, Kyle RA. Retrospective cohort study of 148 patients with polyclonal gammopathy. Mayo Clin Proc 2001;76:476-87.

10. Hsu CW, Lin CH, Yang TL, Chang HT. Splenic inflammatory pseudotumor mimicking angiosarcoma. World J Gastroenterol 2008;14:6421-4.

11. Ge R, Liu C, Yin X, et al. Clinicopathologic characteristics of inflammatory pseudotumour-like follicular dendritic cell sarcoma. Int J Clin Exp Pathol 2014;7:2421-9.

12. Choe JY, Go H, Jeon YK, et al. Inflammatory pseudotumor-like follicular dendritic cell sarcoma of the spleen: a report of six cases with increased IgG4-positive plasma cells. Pathol Int 2013;63:245-51. 